\title{
CONTINUOUS FUNCTIONS INDUCED BY SHAPE MORPHISMS ${ }^{1}$
}

\author{
JAMES KEESLING
}

\begin{abstract}
Let $C$ denote the category of compact Hausdorff spaces and continuous maps and $H: C \rightarrow H C$ the homotopy functor to the homotopy category. Let $S: C \rightarrow S C$ denote the functor of shape in the sense of Holsztyński for the projection functor $H$. Every continuous mapping $f$ between spaces gives rise to a shape morphism $S(f)$ in $S C$, but not every shape morphism is in the image of $S$. In this paper it is shown that if $X$ is a continuum with $x \in X$ and $A$ is a compact connected abelian topological group, then if $F$ is a shape morphism from $X$ to $A$, then there is a continuous map $f: X \rightarrow A$ such that $f(x)=0$ and $S(f)=F$. It is also shown that if $f, g: X \rightarrow A$ are continuous with $f(x)=g(x)=0$ and $S(f)=S(g)$, then $f$ and $g$ are homotopic. These results are then used to show that there are shape classes of continua containing no locally connected continua and no arcwise connected continua. Some other applications to shape theory are given also.
\end{abstract}

Introduction. Let $C$ denote the category of compact Hausdorff spaces and continuous maps and $H: C \rightarrow H C$ the homotopy functor to the homotopy category. Let $S: C \rightarrow S C$ denote the functor of shape in the sense of Holsztyński for the projection functor $H$ [5]. Let $X$ and $Y$ be compact Hausdorff spaces. In [6] it is shown that if $X$ and $Y$ are associated with ANR-systems $\underline{X}$ and $\underline{Y}$, respectively, then there is one to one correspondence between $\operatorname{Mor}_{S C}(X, Y)$ and the homotopy classes of maps of ANRsystems used in the approach of Mardesič and Segal [7]. Thus, our results in this paper will apply to either approach to shape.

In the first part of the paper we show that if $X$ is a continuum with $x \in X$ and $A$ is a compact connected abelian topological group, then if $F \in \operatorname{Mor}_{S C}(X, A)$, then there is a continuous $f: X \rightarrow A$ with $S(f)=F$ and with $f(x)=0$. It is also shown that if $X$ and $A$ are as above and $f, g: X \rightarrow A$ are continuous with $f(x)=g(x)=0$ and with $S(f)=S(g)$, then $f$ and $g$ are homotopic. These results are clearly related to the results in [6].

Received by the editors November 14, 1972.

AMS (MOS) subject classifications (1970). Primary 55D99; Secondary 22B99.

Key words and phrases. Shape functor, homotopy functor, shape domination, compact connected abelian topological group, locally connected continuum, arcwise connected continuum.

1 This research was supported by NSF Grant GP-24616A\#1. 
In the second part of the paper we give applications of these results. It is shown that if $A$ is a compact connected abelian topological group, then if $A$ is not locally connected, and if $X$ shape dominates $A$, then $X$ is not locally connected. It is also shown that if $F \in \operatorname{Mor}_{S C}(G, A)$ where $G$ is a compact connected topological group, then there is a unique continuous homomorphism $f: G \rightarrow A$ with $S(f)=F$.

Notation. We assume the notation and results of [6]. As in that paper it is assumed that the reader is familiar with the approach of Holsztyński to shape theory [5] and the approach of Mardesič and Segal [7]. A knowledge of topological groups is assumed. Pontrjagin [8] is a good reference. We will use the fact shown in [1] that if $G$ is a $T_{1}$ topological group and $L$ is a subgroup of $G$ which is a compact Lie group, then the quotient map $p: G \rightarrow G / L$ is a fibration. Now $p$ can be thought of as the composition of two maps, $p_{0}: G \rightarrow G / L_{0}$, where $L_{0}$ is the component of the identity $e$ in $L$, and $p_{1}: G / L_{0} \rightarrow G / L$. The map $p_{0}$ is monotone and $p_{1}$ is a covering map which is finite-to-one.

The cardinals are thought of as a subset of the ordinals as initial ordinals in the usual way. Infinite cardinals are denoted by $\omega_{\alpha}$ where $\alpha$ is an ordinal and $\omega_{\alpha}$ is the $\alpha$ th infinite cardinal. Thus, $\omega_{0}$ is the first infinite cardinal, $\omega_{1}$ is the first uncountable cardinal, and so on. If $X$ is a topological space, then the weight of $X, w(X)$, is the minimum cardinality of a basis for $X$.

1. Induced mappings. Our main theorems are proved in this section. Basically we show that there is a one-to-one correspondence between $\operatorname{Mor}_{S C}(X, A)$ and $\left\{H(f): f \in \operatorname{Mor}_{C}(X, A)\right.$ and $\left.f(x)=0\right\} \subset \operatorname{Mor}_{H C}(X, A)$ where $X$ is a continuum with $x \in X$ and $A$ is a compact connected abelian topological group. More precisely, Theorems 1.1 and 1.2 taken together imply that if $F: H C \rightarrow S C$ is the functor such that $S=F \circ H$, then for $f, g \in \operatorname{Mor}_{C}(X, A)$, then $S(f)=S(g)$ if and only if $H\left(L_{-f(x)} \circ f\right)=$ $H\left(L_{-g(x)} \circ g\right)$ where $L_{a}: A \rightarrow A$ is left translation by $a \in A$.

1.1. Theorem. Let $F$ be a morphism in SC from $X$ to $A$ where $X$ is a continuum with $x \in X$ and $A$ is a compact connected abelian topological group. Then there is a continuous funciion $f: X \rightarrow A$ with $f(x)=0$ and with $S(f)=F$.

1.2. Theorem. Suppose that $X$ and $A$ are as in Theorem 1.1. Then if $f, g: X \rightarrow A$ are continuous with $f(x)=g(x)=0$, then $S(f)=S(g)$ implies $H(f)=H(g)$.

The key to the proofs of these theorems is Theorem 68 of $[8$, p. 326] which we state before beginning the proofs of the above theorems. 
1.3. Definition. Let $\mathscr{G}=\left\{G_{\alpha} ; \pi_{\alpha \beta} ; \alpha \leqq \beta<\gamma\right\}$ be an inverse system of compact topological groups with $\pi_{\alpha \beta}\left(G_{\beta}\right)=G_{\alpha}$ continuous homomorphisms. Assume that the indexing set is the ordinals $[0, \gamma)$ with the usual ordering. Then $\mathscr{G}$ is a Lie series provided that (1) $G_{0}$ is a Lie group; (2) $\operatorname{ker} \pi_{\alpha \alpha+1}$ is a Lie group for all $\alpha<\gamma$; and (3) for every limit ordinal $\beta<\gamma$, $\bigcap_{\alpha<\beta} \operatorname{ker} \pi_{\alpha \beta}=\{e\}$ in $G_{\beta}$.

1.4. Theorem. [8, Theorem 68, p. 326]. Suppose that $G$ is a compact topological group with $w(G)=\omega_{\gamma}$. Then there is a Lie series $\left\{G_{\alpha} ; \pi_{\alpha \beta}\right.$; $\left.\alpha \leqq \beta<\omega_{\gamma}\right\}$ having $G$ as inverse limit.

Observe that if $w(G)>\omega_{0}$, then $w\left(G_{\alpha}\right)<w(G)$ for all $\alpha<\omega_{\gamma}$ in the Lie series. It will be convenient to prove Theorem 1.2 first and then prove Theorem 1.1.

Proof of Theorem 1.2. Clearly Theorem 1.2 is true if $A$ is a torus, since $A$ is then an ANR and any two functions having the same. shape are homotopic. Now suppose that $w(A)=\omega_{\gamma}$ and let $\left\{A_{\alpha} ; \pi_{\alpha \beta} ; a \leqq \beta<\omega_{\gamma}\right\}$ be a Lie series having $A$ as inverse limit. Of course, all $A_{\alpha}$ 's are abelian in this case since $A$ is abelian. Note that for all $\alpha<\omega_{\gamma}, S\left(\pi_{\alpha} \circ f\right)=S\left(\pi_{\alpha} \circ g\right)$ and $\pi_{\alpha} \circ f(x)=\pi_{\alpha} \circ g(x)=0$ in $A_{\alpha}$ where $\pi_{\alpha}: A \rightarrow A_{\alpha}$ are the projection maps making $A$ the inverse limit of the Lie series. Now $\pi_{0} \circ f$ and $\pi_{0} \circ g$ are homotopic by the above remark. Let $J_{0}: X \times I \rightarrow A_{0}$ be a homotopy with $J_{0} \mid X \times\{0\} \equiv \pi_{0} \circ f$ and $J_{0} \mid X \times\{1\} \equiv \pi_{0} \circ g$ such that $J_{0}(\{x\} \times I)=0 \in A_{0}$. Suppose now that for $0<\beta<\omega_{\gamma}$ we have homotopies $J_{\alpha}: X \times I \rightarrow A_{\alpha}$ defined for all $\alpha<\beta$ such that (1) $J_{\alpha} \mid X \times\{0\} \equiv \pi_{\alpha} \circ f$ and $J_{\alpha} \mid X \times\{1\} \equiv \pi_{\alpha} \circ g$; (2) such that $J_{\alpha}(\{x\} \times I)=0 \in A_{\alpha}$; and (3) such that for all $\alpha \leqq \delta<\beta$, $J_{\alpha}=\pi_{\alpha \delta} \circ J_{\delta}$. Then if $\beta$ is a limit ordinal, then let $J_{\beta}$ be the limit of the maps $J_{\alpha}: X \times I \rightarrow A_{\alpha}$ in the inverse system $\left\{A_{\alpha} ; \pi_{\alpha \delta} ; \alpha \leqq \delta<\beta\right\}$. Then $\left\{J_{\alpha}: \alpha \leqq \beta\right\}$ has properties (1), (2), and (3) above. If $\beta=\alpha+1$, then let $\pi_{\alpha \alpha+1}=p \circ q$ where $q: A_{\alpha+1} \rightarrow A^{\prime}$ is a monotone homomorphism and $p: A^{\prime} \rightarrow A_{\alpha}$ is a finiteto-one covering map so that $p \circ q$ is the monotone-light factorization of $\pi_{\alpha \alpha+1}$. Note that the kernel of $q$ is a torus in $A_{\alpha+1}$.

Claim. Let $B$ be a subgroup of $A$ with $A$ a compact connected abelian topological group and $B$ a torus. Then $A \cong B \times A / B$.

Proof of Claim. Let $e: B \rightarrow A$ denote the imbedding of $B$ as a subgroup. Then $e^{*}$ : char $A \rightarrow \operatorname{char} B$ is onto. Now char $B=Z^{n}$ is free. Thus char $A \cong Z^{n} \times \operatorname{ker} e^{*}$. Thus $A \cong B \times C$ where $C=$ charker $e^{*}$. Clearly $C \cong A / B$.

The claim allows us to view $A_{\alpha+1}$ as $A^{\prime} \times \operatorname{ker} q$. Since $p$ is a covering map, there is a unique lifting of $J_{\alpha}$ to $J^{\prime}: X \times I \rightarrow A^{\prime}$ such that $J^{\prime}(\{x\} \times I)=$ $0 \in A^{\prime}$ and $J^{\prime} \mid X \times\{0\}=q \circ \pi_{\alpha+1} \circ f$ and $J^{\prime} \mid X \times\{1\}=q \circ \pi_{\alpha+1} \circ g$. Let $r: A_{\alpha+1} \rightarrow$ ker $q$ be the projection map. Since ker $q$ is a torus and $S\left(r \circ \pi_{\alpha+1} \circ f\right)=$ $S\left(r \circ \pi_{\alpha+1} \circ g\right)$ with $r \circ \pi_{\alpha+1} \circ f(x)=r \circ \pi_{\alpha+1} \circ g(x)=0 \in \operatorname{ker} q$, there is a 
homotopy $J^{\prime \prime}: X \times I \rightarrow \operatorname{ker} q$ such that $J^{\prime \prime} \mid X \times\{0\}=r \circ \pi_{\alpha+1} \circ f$ and $J^{\prime \prime} \mid X \times$ $\{1\}=r \circ \pi_{\alpha+1} \circ g$ with $J^{\prime \prime}(\{x\} \times I)=0 \in \operatorname{ker} q$. Then let $J_{\alpha+1}: X \times I \rightarrow A_{\alpha+1}$ be defined by $J_{\alpha+1}(x, t)=\left(J^{\prime}(x, t), J^{\prime \prime}(x, t)\right) \in A^{\prime} \times \operatorname{ker} q=A_{\alpha+1}$. Then in this case also $\left\{J_{\alpha}: \alpha \leqq \beta\right\}$ has properties (1), (2), and (3) above. Proceeding by transfinite induction we get a collection of maps $\left\{J_{\alpha}: \alpha<\omega_{\gamma}\right\}$ satisfying properties (1), (2), and (3). Then letting $J: X \times I \rightarrow A$ be the limit of $\left\{J_{\alpha}: \alpha<\omega_{\gamma}\right\} J$ will be a homotopy from $f$ to $g$ and Theorem 1.2 is proved.

ProOF OF THEOREM 1.1. The proof of Theorem 1.1 proceeds by transfinite induction on $w(A)$. If $A$ is a torus, then clearly there is such a continuous $f: X \rightarrow A$ since $A$ is an ANR. We can require $f(x)=0$ by using left multiplication in $A$. Suppose now that $w(A)=\omega_{0}$. Then let $A$ be the inverse limit of a sequence of Lie groups $\left\{A_{i} ; \pi_{i j} ; i \leqq j<\omega_{0}\right\}$. Of course, the $A_{i}$ 's are tori in this case. By [1], each map $\pi_{i+1}$ is a fibration. By the remarks above, $S\left(\pi_{i}\right) \circ F=S\left(f_{i}\right)$ for some $f_{i}: X \rightarrow A_{i}$ with $f_{i}(x)=0$ for each $i$ where $\pi_{i}: A \rightarrow A_{i}$ are the projection maps making $A$ the limit of $\left\{A_{i}\right\}$. Let $g_{1}=f_{1}$. Now $S\left(g_{1}\right)=S\left(\pi_{1}\right) \circ F=S\left(\pi_{12}\right) \circ S\left(\pi_{2}\right) \circ F=S\left(\pi_{12} \circ f_{2}\right)$. By Theorem 1.2, $g_{1}$ and $\pi_{12} \circ f_{2}$ are homotopic. Since $\pi_{12}$ is a fibration the map $g_{1}$ can be lifted to a map $g_{2}$ such that $g_{2}$ is homotopic to $f_{2}$ with $g_{2}(x)=0$. By the same argument $g_{2}$ can be lifted to a map $g_{3}: X \rightarrow A_{3}$ such that $g_{3}$ is homotopic to $f_{3}$ with $g_{3}(x)=0$. Proceeding by induction we get a sequence of maps $g_{i}: X \rightarrow A_{i}$ with $g_{i}(x)=0$ and with $g_{i}=\pi_{i j} \circ g_{j}$ for all $i \leqq j<\omega_{0}$ with $f_{i}$ and $g_{i}$ homotopic. Thus $S\left(g_{i}\right)=S\left(\pi_{i}\right) \circ F$. Let $f: X \rightarrow A$ be the limit of the maps $\left\{g_{i}\right\}$. Then $S(f)=F$ by the continuity of the shape functor [5]. Clearly $f(x)=0$.

Now suppose that $w(A)=\omega_{\gamma}>\omega_{0}$ and assume that for all $\omega_{\alpha}<\omega_{\gamma}$ if $w(A)=\omega_{\alpha}$, then the theorem is true for $A$. Then let $\left\{A_{\alpha} ; \pi_{\alpha \beta} ; \alpha \leqq \beta<\omega_{\gamma}\right\}$ be a Lie series having $A$ as inverse limit. Note that $w\left(A_{\alpha}\right)<\omega_{\gamma}$ for all $\alpha<\omega_{\gamma}$. Thus there are maps $f_{\alpha}: X \rightarrow A_{\alpha}$ with $f_{\alpha}(x)=0 \in A_{\alpha}$ and $S\left(f_{\alpha}\right)=$ $S\left(\pi_{\alpha}\right) \circ F$ for all $\alpha<\omega_{\gamma}$. Let $g_{0}=f_{0}$ and suppose that $g_{\alpha}$ has been defined for all $\alpha<\beta<\omega_{\gamma}$ such that $g_{\alpha}: X \rightarrow A_{\alpha}$ is homotopic to $f_{\alpha}$ with $g_{\alpha}(x)=0 \in A_{\alpha}$ and with $g_{\alpha}=\pi_{\alpha \delta} \circ g_{\delta}$ for all $\alpha \leqq \delta<\beta$. Then if $\beta$ is a limit ordinal let $g_{\beta}: X \rightarrow A_{\beta}$ be the limit of the maps $\left\{g_{\alpha}: \alpha<\beta\right\}$. Then $g_{\beta}(x)=0$ and $S\left(g_{\beta}\right)=$ $S\left(\pi_{\beta}\right) \circ F=S\left(f_{\beta}\right)$ by the continuity of the shape functor. Thus by Theorem $1.2, f_{\beta}$ and $g_{\beta}$ are homotopic. Now if $\beta=\alpha+1$, then by [1], $\pi_{\alpha \alpha+1}$ is a fibration with $g_{\alpha}$ homotopic to $\pi_{\alpha \beta} \circ f_{\beta}$. Thus $g_{\alpha}$ can be lifted to a map $g_{\beta}$ homotopic to $f_{\beta}$ with $g_{\beta}(x)=0$. Thus proceeding by transfinite induction we obtain a collection of maps $\left\{g_{\alpha}: \alpha<\omega_{\gamma}\right\}$ with $g_{\alpha}: X \rightarrow A_{\alpha} ; g_{\alpha}(x)=0$; and $S\left(g_{\alpha}\right)=S\left(\pi_{\alpha}\right) \circ F$. Letting $f$ be the limit of these maps, we get that $f(x)=0$ and $S(f)=F$. Thus the theorem is true for $w(A)=\omega_{\gamma}$. Theorem 1.1 now follows by transfinite induction on $w(A)$.

It is not known if Theorems 1.1 and 1.2 would be true for $A$ an 
arbitrary compact connected topological group. However, Theorem 1.1 can be shown for this case if $w(A)=\omega_{0}$.

1.5. Theorem. Let $X$ be a continuum and $x \in X$. Let $A$ be a compact connected topological group with identity element $e$ and with $w(A)=\omega_{0}$. Then, if $F \in \operatorname{Mor}_{S C}(X, A)$, there is a continuous map $f: X \rightarrow A$ with $f(x)=e$ and with $S(f)=F$.

Proof. Let $\left\{A_{i} ; \pi_{i j} ; i \leqq j<\omega_{0}\right\}$ be a sequence of Lie groups having $A$ as inverse limit with the projection maps being $\pi_{i}: A \rightarrow A_{i}$. For each $i$, there is a continuous map $f_{i}: X \rightarrow A_{i}$ with $f(x)=e \in A_{i}$ such that $S\left(f_{i}\right)=$ $S\left(\pi_{i}\right) \circ F$ since each $A_{i}$ is an ANR. Using the fact that $\pi_{i i+1}$ is a fibration for each $i$ and that $f_{i}$ is homotopic to $\pi_{i+1} \circ f_{i+1}$ for each $i$, construct $g_{i}: X \rightarrow A_{i}$ with $g_{i}(x)=e \in A_{i}$ with $g_{i}$ homotopic to $f_{i}$ and with $g_{i}=\pi_{i j} \circ g_{j}$ for all $i \leqq j<\omega_{0}$. Then let $f$ be the limit of the $g_{i}$ 's. Then $S(f)=F$ by the continuity of the shape functor.

2. Applications. In this section we apply Theorem 1.1 to obtain several theorems and examples in shape theory.

2.1. THEOREM. Let $A$ be a compact connected abelian topological group and $X$ a continuum. Suppose that $S(X) \geqq S(A)$. Then there is a continuous map $f: X \rightarrow A$ such that $f(X)=A$.

Proof. Suppose that $S(X) \geqq S(A)$. Then there are shape morphisms $F: X \rightarrow A$ and $G: A \rightarrow X$ such that $F \circ G=1_{A}$ in $S C$. By Theorem 1.1 there is a continuous map $f: X \rightarrow A$ such that $S(f)=F$. We will now show that $f(X)=A$. Suppose that $f(X) \neq A$. Let $e: f(X) \rightarrow A$ be the inclusion map. Now $A$ is a limit manifold $\left[3,2.12\right.$, p. 345]. Thus $e^{*}: H^{*}(A) \rightarrow H^{*}(f(X))$ is not a monomorphism (the proof of Proposition 2.11 in [3, p. 344]). But then $f^{*}: H^{*}(A) \rightarrow H^{*}(X)$ cannot be a monomorphism either. But then $G^{*} \circ F^{*}=G^{*} \circ f^{*}: H^{*}(A) \rightarrow H^{*}(A)$ cannot be $1_{H^{*}(A)}$, a contradiction. Thus $f(X)=A$ as asserted.

2.2. Corollary. Let $A$ be a compact connected abelian topological group. Let $X$ be a continuum. If $S(X) \geqq S(A)$ and $X$ is locally connected, then $A$ is locally connected. If $S(X) \geqq S(A)$ and $X$ is arcwise connected, then $A$ is arcwise connected.

Actually Corollary 2.2 is true for any property $\mathscr{P}$ such that if $X$ has property $\mathscr{P}$ and $f(X)=Y$ is a quotient map, then $Y$ has property $\mathscr{P}$.

2.3. Example. Let $\Sigma_{a}$ be a solenoid which is not a circle. Then for any continuum $X$ with $S(X) \geqq S\left(\Sigma_{a}\right), X$ cannot be arcwise connected or locally connected. Actually there must be at least $2^{\omega_{0}}$ arccomponents of 
$X$ because of the map $f(X)=\Sigma_{a}$. Borsuk [0] proved that no (metric) Peano continuum can shape dominate a solenoid.

2.4. EXAMPLE. Let $Z$ be the integers and let $A=\operatorname{char} Z^{\omega_{0}}$. It is known that $A$ is a locally connected connected abelian topological group which is not arcwise connected. For a proof of this last fact see [2, 4.82, p. 194]. The space $A$ is an example of a locally connected continuum such that no arcwise connected continuum shape dominates $A$. In particular, no arcwise connected continuum has the same shape as $A$. This was mentioned without proof in [6]. In [6] it was shown that $A$ is movable since it is locally connected.

2.5. THEOREM. Let $G$ be a compact connected topological group and $A$ a compact connected abelian topological group. Then if $F \in \operatorname{Mor}_{S C}(G, A)$, then there is a unique continuous homomorphism $f: G \rightarrow A$ such that $S(f)=F$.

Proof. Let $F \in \operatorname{Mor}_{S C}(G, A)$. Then by Theorem 1.l, there is a continuous $g: G \rightarrow A$ such that $g(e)=0$ with $S(g)=F$. By [9], $g$ is homotopic to a continuous homomorphism $f: G \rightarrow A$. Thus $S(f)=F$ also. If $f^{\prime}: G \rightarrow A$ is a continuous homomorphism with $S\left(f^{\prime}\right)=F$, then $f^{\prime}$ and $f$ are homotopic by Theorem 1.2. But by [9], $f^{\prime}=f$ and Theorem 2.5 is proved.

Theorem 2.5 generalizes Theorem 1.2 in [6].

\section{REFERENCES}

0. K. Borsuk, On a locally connected non-movable continuum, Bull. Acad. Polon. Sci. Sér. Sci. Math. Astronom. Phys. 17 (1969), 425-430. MR 41 \#1012.

1. A. M. Gleason, Spaces with a compact Lie group of transformations, Proc. Amer. Math. Soc. 1 (1950), 35-43. MR 11, 497.

2. K. H. Hofmann, Introduction to the theory of compact groups. I, Tulane University Lecture Notes, Tulane University, New Orleans, La., 1968.

3. K. H. Hofmann and P. S. Mostert, Elements of compact semigroups, Charles E. Merrill Books, Columbus, Ohio, 1966. MR 35 \#285.

4. W. Holsztyński, Continuity of Borsuk's shape functor, Bull. Acad. Polon. Sci. Sér. Sci. Math. Astronom. Phys. 19 (1971), 1105-1108.

5. - - An extension and axiomatic characterization of Borsuk's theory of shape, Fund. Math. 70 (1971), no. 2, 157-168. MR 43 \#8080.

6. J. Keesling, Shape theory and compact connected abelian topological groups, Trans. Amer. Math. Soc. (to appear).

7. S. Mardesič and J. Segal, Shapes of compacta and ANR-systems, Fund Math. 72 (1971), 41-59.

8. L. S. Pontrjagin, Topological groups, 2nd ed., GITTL, Moscow, 1954; English transl., Gordon and Breach, New York, 1966. MR 17, 171; 34 \#1439.

9. W. Scheffer, Maps between topological groups that are homotopic to homomorphisms, Proc. Amer. Math. Soc. 33 (1972), 562-567.

10. E. Spanier, Algebraic topology, McGraw-Hill, New York, 1966. MR 35 \#1007.

Department of Mathematics, University of Florida, Gainesville, Florida 32601 . 